\title{
Energy Consumption Analysis of Programs based on XMOS ISA-Level Models
}

\author{
S. Kerrison ${ }^{1}$, U. Liqat ${ }^{2}$, K. Georgiou ${ }^{1}$, A. Serrano ${ }^{2}$, N. Grech ${ }^{1}$, \\ P. Lopez-Garcia ${ }^{2,3}$, K. Eder ${ }^{1}$, and M.V. Hermenegildo ${ }^{2,4}$ * \\ 1 University of Bristol \\ 2 IMDEA Software Institute \\ ${ }^{3}$ Spanish Council for Scientific Research (CSIC) \\ ${ }^{4}$ Universidad Politécnica de Madrid (UPM)
}

\begin{abstract}
Energy consumption analysis of embedded programs necessitates the analysis of low-level program representations. This is challenging because the gap between the high-level program structure and the low-level energy models needs to be bridged. Here, we describe techniques for recreating the structure of low-level programs and transforming these into Horn clauses in order to make use of the CiaoPP resource analysis framework. Our analysis framework, which makes use of an energy model we produce for the underlying hardware, characterizes the energy consumption of the program, and returns energy formulae parametrised by the size of the input data. We have performed an initial experimental assessment and obtained encouraging results when comparing the statically inferred formulae to direct energy measurements from the hardware running a set of benchmarks. Static energy estimation has applications in program optimization and enables more energy-awareness in software development.
\end{abstract}

Keywords: energy consumption analysis, energy models, resource usage analysis, static analysis.

\section{Introduction}

Energy consumption and the environmental impact of computing technologies are a major focus. Despite advances in power-efficient hardware, more energy savings can be achieved by improving the way current software technologies make use of such hardware. Many optimization techniques that can be used for producing energy-efficient software need estimations of the energy consumption of software segments prior to their execution, in order to make decisions about the optimal way of executing them. These a priori estimations are also very useful to software engineers to better understand the effect of their designs on the

\footnotetext{
* The research leading to these results has received funding from the European Union 7th Framework Programme under grant agreement no 318337, ENTRA - WholeSystems Energy Transparency, Spanish MINECO TIN'12-39391 StrongSoft and TIN'08-05624 DOVES projects, and Madrid TIC/1465 PROMETIDOS-CM project.
} 
energy consumption early on during the software development process, and make more informed design decisions (e.g., using the appropriate data structures), even when there are parts not developed yet.

In this paper we propose a novel (automatic) static analysis capable of estimating the energy consumption of an embedded program (and its constituent parts, such as procedures and functions) as a function on several parameters of the input data (e.g., sizes), and the hardware platform where they are executed (e.g., clock frequency and voltage). We show the feasibility of our proposal with a concrete case study: analysis of ISA (Instruction Set Architecture) code compiled from XC [14]. XC is a high-level C-based programming language that includes extensions for concurrency, communication, input/output operations, and realtime behavior. XC libraries share a common API with standard $\mathrm{C}$ libraries and therefore $\mathrm{C}$ code can commingle with $\mathrm{XC}$ code in a single application.

Since energy resource analysis depends on the underlying hardware, the analyser requires information expressing the effect of the execution of a software segment (e.g., an assembly instruction) on the hardware. Such information is represented using models. In our approach these models express information using assertions, which is propagated during the static analysis process in order to infer information for higher-level entities such as functions. For instance, using assertions we abstract the operations in the language in terms of their effect on the size of the runtime data and the energy exerted. Energy models at lower levels (e.g., at the ISA level) are more precise than at higher levels (e.g., XC source code), since the closer to the hardware, the easier it is to determine the effect of the execution of the program on the hardware. For this reason, we have produced models for the ISA level, which we use when analysing ISA code generated by the XCC compiler.

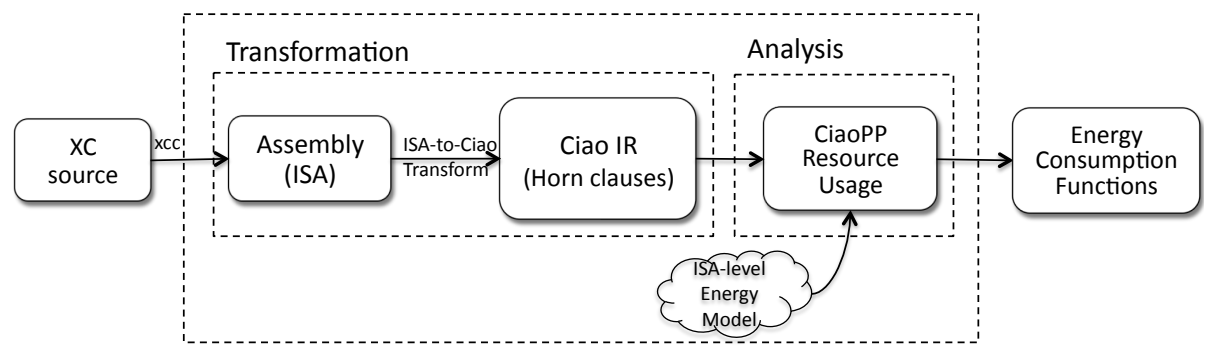

Fig. 1. Overview of the analysis framework for XC programs.

Our approach leverages the CiaoPP tool [5], the preprocessor of the Ciao programming environment [6]. CiaoPP includes a parametric analysis framework for resource usage that can be instantiated to infer resources of interest, energy consumption in our case. This instantiation is done by means of an assertion language that allows the user to define resources and express the resource usage of elementary program operations, certain program constructs, and library procedures. Based on this information, the analyser can infer upper and lower bounds on the resource usage of the whole program. This CiaoPP analysis works on an intermediate block-based representation language, which we call the Ciao 
IR. Each block is represented as a Horn clause, so that, in essence, the Ciao IR is a logic programming subset of the Ciao language. To this end we propose a transformation of the ISA program into Ciao IR (containing Horn clauses and assertions), which allows us to analyse the transformed program with CiaoPP. The procedural interpretation of these Ciao IR programs, coupled with resourcerelated information contained in the assertions (such as the energy consumption models at the ISA level), allow the resource analysis to infer static bounds on the energy consumption of the Ciao programs that are applicable to the original ISA programs.

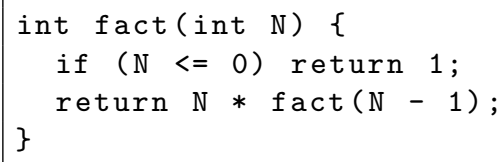

Fig. 2. An XC source (factorial) function.

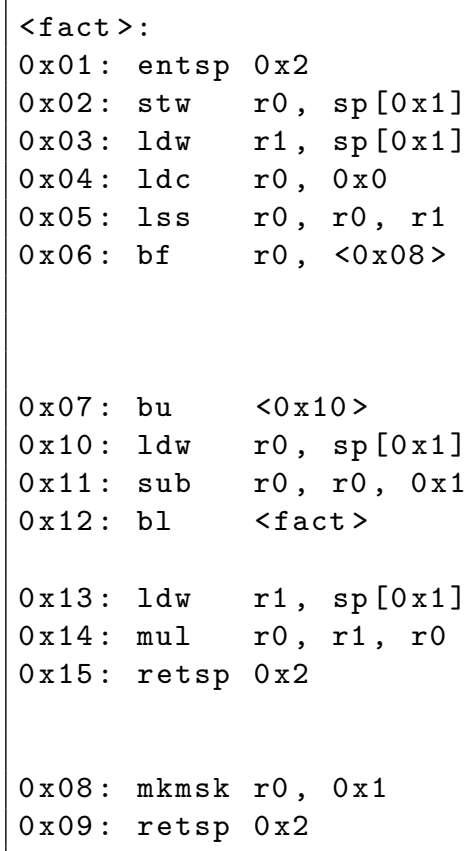

Fig. 3. An ISA (factorial) program (left side) and its Ciao IR (right side).

Figure 1 shows the main steps of our approach for energy consumption analysis, which starts with an XC program (e.g., the fact function in Figure 2). The ISA program corresponding to it is generated using the $\mathrm{XC}$ compiler tool $\mathrm{XCC}$ (left hand side of Figure 3). The resulting ISA program is passed to a translator which generates the associated Ciao IR program (right hand side of Figure 3). Such program, together with the information contained in the energy models at 
the ISA level (represented using the mentioned assertion language), is passed to the resource analysis which outputs the energy consumption for all procedures in the Ciao IR program. In our example, the resource analysis infers an estimation of the energy consumed by a call to fact as $(26.0 N+19.4) n J$. This is parametric with $N$, the input argument to fact.

In the rest of the paper, energy characterization and modelling for our case study architecture (XMOS XS1-L) is explained in Section 2. Then, Section 3 describes the translation from ISA programs into Ciao IR, and Section 4 the instantiation of the CiaoPP general resource usage analysis framework to infer energy consumption. In Section 5, we have performed an experimental assessment of our approach, showing that the estimation of energy consumption is reasonably accurate. Section 6 comments on related work. Finally, Section 7 summarises our conclusions and comments on ongoing and future work.

\section{Energy Characterization and Modelling}

The assertion-based model utilises power consumption data collected during hardware measurement. We have developed an ISA-level model that provides software energy consumption estimates based on Instruction Set Simulation (ISS) statistics. The hardware, the measurement process, as well as the construction of the ISS-driven model, are detailed in [3], with the key components relevant to this paper explained in the rest of this section.

The practicality and accuracy of our approach to energy consumption analysis relies on a good characterization of energy consumption and generating good energy consumption models. A trade-off needs to be found between the simplicity of the models, which improves the efficiency of the analysis, and the accuracy of the models, which improves the accuracy of the global analysis. In this paper we describe an initial proposal that offers a good compromise.

\subsection{Energy profiling framework and strategy}

An energy profiling framework, xmprofile, is used to generate sequences of instructions under various constraints in order to profile the energy characteristics of the hardware. This data is essential for the accurate application of any models, be they at the ISS level, or at a higher level utilising static analysis.

The hardware used by this framework is shown in Figure 4. A master processor communicates with a current measurement circuit for the power supply to the core of a slave processor, the Device Under Test (DUT). A series of tests are loaded onto the slave processor and power samples are taken during each test.

The multi-threaded architecture of the XS1-L1 processor requires that tests be tightly coupled in order to ensure the progression of specific combinations of instructions through the pipeline. This is due to each instruction in the pipeline being from a neighbouring thread, rather than the next instruction as would typically be the case in a sequential embedded processor. By utilising the device's thread synchronisation capabilities, combined with unrolled test loops, a large number of combinations of instructions and data values can be tested and measured. 


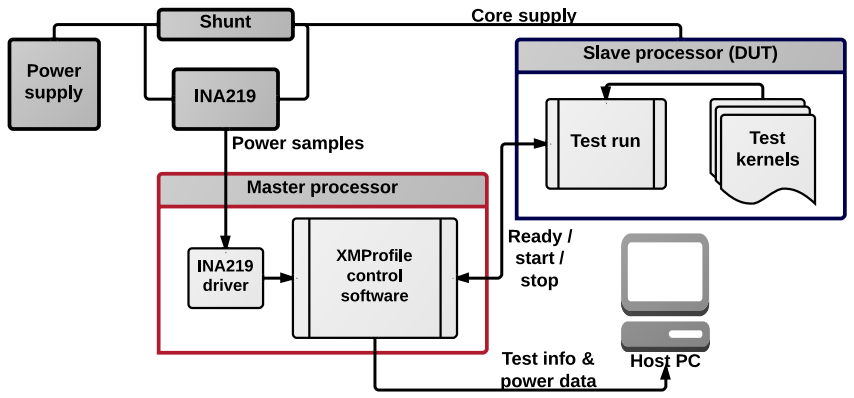

Fig. 4. Overview of test harness hardware and software structure, with a slave processor executing test kernels and a master processor collecting power samples.

Currently, a subset of the ISA, including arithmetic operations, logic operations, and condition tests, has been characterized. Other instructions are at the moment approximated using an average value, based on typical observed behaviour.

\subsection{ISA-level model}

An ISA-level model, xmmodel, gives an energy estimate for a program based on ISS output. Data from the measurement framework feeds this model.

Our model is based on that devised by Tiwari [13]. Tiwari's approach is shown in Equation 1. The energy of a program, $E_{p}$, is characterised as the sum of base energy cost, $B_{i}$, for all ISA instructions, $i$, multiplied by the number of executions of each instruction, $N_{i}$. An inter-instruction overhead energy, $O_{i, j}$, is then accounted for by enumerating for all instruction combinations $i, j$ and their frequency, $N_{i, j}$. Finally, additional contributions to program energy can be accounted for by $k$ external effects, $E_{k}$, which may include externally modelled behaviours such as cache memory.

$$
E_{p}=\sum_{i \in \mathrm{ISA}}\left(B_{i} \times N_{i}\right)+\sum_{i, j \in \mathrm{ISA}}\left(O_{i, j} \times N_{i, j}\right)+\sum_{k \in \mathrm{ext}} E_{k}
$$

The XS1 architecture is hardware multi-threaded. This necessitates a fundamental revision of the model equation. In addition, for performance reasons, the ISS collects instruction statistics rather than a full trace. This reduces the execution time by an order of magnitude, such that it is approximately 100 times slower than the hardware when simulation is run on a modern computer.

Equation 2 describes the energy of a program, $E_{p}$, using a similar method to Equation 1, but with several key differences. Time is an explicit component, multiplied by power terms in order to calculate energy. This separation enables future exploration of idle periods, external event timing, and variable operating frequencies. Inter-instruction overhead is represented as a single component, rather than considering it for all possible pairs, on account of a statistics-based approach rather than cycle-by-cycle instruction tracing. Finally, the level of concurrency must be accounted for, something that was not necessary for the ar- 
chitecture targeted by Equation 1.

$$
E_{\mathrm{p}}=P_{\text {base }} N_{\text {idle }} T_{\text {clk }}+\sum_{t=1}^{N_{t}} \sum_{i \in \mathrm{ISA}}\left(\left(M_{t} P_{i} O+P_{\text {base }}\right) N_{i, t} T_{\text {clk }}\right)
$$

The base power, $P_{\text {base }}$, is used both in periods of activity and during idle. The number of idle periods, $N_{\text {idle }}$, is counted and multiplied by the clock period, $T_{c l k}$, to account for the energy consumed when no threads are active. For each number of concurrent threads, $t$, (based on the proportion of time each thread is active), and for each instruction, $i$, in the ISA, the instruction power, $P_{i}$, is multiplied by a constant inter-instruction power overhead, $O$, and a concurrency cost for the level of concurrency at which the processor is operating, $M_{t}$. These are all multiplied by the number of times this instruction occurs at this concurrency level, $N_{i, t}$, and the clock period. Combined with the idle energy, this gives a total energy estimate for the program run.

In the case where a single thread is running, with no idle periods, then the above can be simplified to Equation 3. The result is very similar to the singlethreaded Tiwari equation, but with only a single, generic inter-instruction power overhead component, $O$, and with no external " $k$ " components as the memory of the XS1-L is single-cycle with no cache, with no other effects that need to be considered at this point. Again, in 3 time is an explicit component.

$$
E_{\mathrm{p}}=\sum_{i \in \mathrm{ISA}}\left(\left(M_{1} P_{i} O+P_{\text {base }}\right) \times\left(N_{i} T_{\text {clk }}\right)\right)
$$

Our ISS-based model will be used as a comparison point between actual hardware energy measurements and the estimations calculated through static analysis. Both approaches rely on the same energy profiling data, so the effect of this on accuracy, as well as the effect of different modelling approaches (i.e. simulation and static analysis), can be evaluated.

\section{Transforming ISA Programs into Ciao IR Programs}

In this section we describe the transformation from ISA programs into the Ciao intermediate representation (Ciao IR) mentioned in Section 1, which is used for analysis. Such representation consists of a sequence of blocks (as in Figure 3). Each block is represented as a Horn clause:

$$
<\text { block_id }>(<\text { params }>):-S_{1}, \ldots, S_{n} .
$$

which has an entry point, that we call the head of the block (to the left of the :- symbol), including a number of parameters $\langle$ params $\rangle$, and a sequence of steps (the body, to the right of the :- symbol), each of which is either, (the representation of) an ISA instruction, or a call to another (or the same) block. The analyzer deals with the Ciao IR always in the same way, independently of its origin. The transformation ensures that the program information relevant to resource usage is preserved, so that the energy consumption functions of the Ciao IR programs inferred by the resource analysis are applicable to the original ISA programs.

ISA programs are expressed using the XS1 instruction set [10]. The transformation framework currently works on a subset of this instruction set. The ISA 
program is parsed and a control flow analysis is carried out, yielding an interprocedural control flow graph (CFG). This process starts by identifying control transfer instructions such as branch or call instructions. Basic blocks are then constructed, which are annotated with input/output arguments and transformed into Static Single Assignment (SSA) form. Finally, the target Ciao IR (i.e., Horn clauses) is emitted.

A basic block over a CFG is a maximal sequence of distinct instructions, $S_{1}$ through $S_{n}$, such that all instructions $S_{k}$ where $1<k<n$ have exactly one inedge and one out-edge (excluding call/return edges), $S_{1}$ has one out-edge, and $S_{n}$ has one in-edge. A basic block therefore has exactly one entry point at $S_{1}$ and one exit point at $S_{n}$. All call instructions are assumed to return. Using the basic block definition a block control flow graph is constructed by the analyser, where each node represents a block. Edges between the blocks are derived from calls/jumps between blocks. This process involves iterating through the CFG of the ISA program and marking block boundaries, which are instructions that either begin or end a basic block.

Inferring block input/output parameters. In order to treat each block as a Horn clause, the block's input and output arguments need to be inferred. For the entry block, the input and output arguments are precisely those of the main function in the program. We define the functions params in $_{\text {and }}$ anams out $_{\text {, }}$, which infer input and output parameters of a block respectively. These are recomputed until a least fixpoint is reached on these functions.

$$
\begin{aligned}
\text { params }_{\text {out }}(b) & =\operatorname{kill}(b) \cup \bigcup_{b^{\prime} \in \text { next }(b)} \text { params }_{\text {out }}\left(b^{\prime}\right) \\
\text { params }_{i n}(b) & =\operatorname{gen}(b) \cup \bigcup_{b^{\prime} \in \operatorname{next}(b)} \text { params }_{\text {in }}\left(b^{\prime}\right)
\end{aligned}
$$

where next $(b)$ denotes the set of immediate target blocks that can be reached from $b$ with a call or jump, while gen $(k)$ and $k i l l(k)$ are the read and written variables in a block respectively, which we define as:

$$
\operatorname{kill}(b)=\bigcup_{k=1}^{n} \operatorname{def}(k), \quad \operatorname{gen}(b)=\bigcup_{k=1}^{n}\{v \mid v \in \operatorname{ref}(k) \wedge \forall(j<k) . v \notin \operatorname{def}(j)\}
$$

and $\operatorname{def}(k)$ and $r e f(k)$ denote the variables written or referred to at a node in the block respectively.

Resolving branching to multiple blocks. In the XS1 instruction set, conditional branch instructions (e.g., bt, bf) jump to one of the two target blocks based on the value of the branching variable. For example, in Figure 3, at line 7 the bf instruction (branch if fail) will jump to address $0 \times 8$ if $r 0=0$, otherwise to address $0 \times 7$. In the Ciao IR this branch needs to be a call to one of the two blocks.

We use a similar approach to the one described in [11] to resolve branches to multiple blocks. The multiple target blocks of a jump instruction are assigned the same head, which essentially are clauses of the same Ciao IR predicate. This is achieved by merging the heads of the target clauses so that each clause has the same head. The algorithm is trivial, since we have already inferred the 
input/output parameters to each block's head. The input/output parameters to the new head of the clauses are the union of the input/output parameters of all the clauses along with the branching variable. This enables preservation of the branching semantics of the original ISA program in Ciao IR form.

For example in Figure 3, the bf instruction at line 7 of the ISA program is changed to a dummy literal at line $7 \mathrm{a}$ in the Ciao IR, plus a predicate call to fact_aux on line $7 \mathrm{~b}$. The predicate fact_aux has two clauses, each representing one of the target blocks of the bf instruction. The dummy literal for the bf instruction is created so that the resource usage analysis can take it into account when inferring energy usage functions.

Static Single Assignment form (SSA). The last step is to convert a block representation into static single assignment (SSA) form, where each variable is assigned exactly once and multiple assignments to the same variable create new versions of that variable.

In compilers, the SSA form is generated at the function level (e.g., at LLVM [9] level) where a function might consist of multiple basic blocks. However, we follow the approach of generating SSA form at the block level, and therefore we do not need to generate $\phi$ nodes. A $\phi$ node is an instruction used to select a version of the variable depending on the predecessor of the current block. Since each block is already annotated with input/output arguments, any predecessor block will pass the appropriate values as input parameters when making a call to the target block.

In Figure 3, the Ciao IR (right hand side) is in SSA form, where each variable is defined exactly once and stack references are transformed to local variables. Each instruction is transformed into a Ciao IR literal with input/output variables.

Analysis on low level (ISA) representations, in general, suffers from the problem of extracting a precise control flow graph in the presence of indirect jumps and calls. The current implementation of our transformation is restricted to direct jumps and calls. We plan to integrate other techniques into the transformation tool to resolve such problems including recognizing code patterns used by compilers and performing static program analysis (see [16] and its references).

\section{General Analysis Framework}

In this section we introduce the CiaoPP general resource usage analysis framework and discuss how to instantiate it for the analysis of the Ciao IR programs resulting from the translation of ISA programs.

CiaoPP includes a global static analyser which is parametric with respect to resources and type of approximation (lower and upper bounds) [12]. The user can define the parameters of the analysis for a particular resource by means of assertions that associate basic cost functions with elementary operations of the base language and procedures in libraries, thus expressing how they affect the usage of a particular resource. The global static analysis can then infer bounds on the resource usage of all the procedures in the program, as functions of input data sizes. Examples of resources that can be analysed by instantiating the 
CiaoPP general framework include execution steps, execution time, number of accesses to a database, number of bytes sent or received through a socket, etc.

In the rest of the section we use a running example to illustrate the main concepts and steps of the analysis framework. In particular, and for simplicity, assume that we are interested in estimating upper bounds on the energy consumed by the Ciao IR program in Figure 3 (right hand side) generated from its $\mathrm{XC}$ code in Figure 2.

\subsection{Instantiating the General Framework}

Defining Resources. We start by defining the identifier ("counter") associated to the energy consumption resource, through the following Ciao declaration:

:- resource energy.

Expressing Energy Models and Resource Usage of Library Functions. The resource usage of Ciao library predicates is expressed using "trust" assertions (see [6] and its references for a description of the Ciao assertion language). For example, we can write assertions for each Ciao predicate that represents an ISA instruction; these constitute the energy models. The following assertions (for the add and sub instructions) are part of the simple energy model that we used in the static analysis for our experiments, which assigns a constant energy amount to each ISA instruction:

:- trust pred add (X,Y,Z) + resource(avg, energy, 1215439).

:- trust pred $\operatorname{sub}(\mathrm{X}, \mathrm{Y}, \mathrm{Z})+$ resource (avg, energy, 1210759).

Note that the first argument (avg) of the resource property (in the global computational properties field "+" of the assertions) expresses that the given energy consumption for the ISA instructions is an average value. Such model is obtained using the measurement process described in Section 2, based on Equation (3), so that the energy cost (given in $f J$ above) for an ISA instruction $i$ is $c_{i}=\left(M_{1} P_{i} O+P_{\text {base }}\right) T_{\text {clk }}$.

Assertions are also used to express information that is instrumental in the resource usage analysis. For example, assertion:

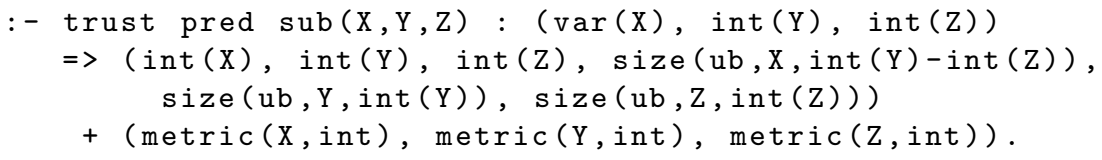

indicates that if the $\operatorname{sub}(\mathrm{X}, \mathrm{Y}, \mathrm{Z})$ predicate (representing the "substraction" ISA instruction) is called with $\mathrm{X}$ and $\mathrm{Y}$ bound to integer numbers and $\mathrm{Z}$ an unbound variable (precondition field ":"), after the successful completion of the call (postcondition field "=>"), $\mathrm{X}$ is an integer number whose size is the size of Y minus the size of $Z$. It also expresses that the size metric used for the three arguments is "int", the actual value of the integer numbers. 


\subsection{Performing the Analysis}

Once the parameters of the general resource analysis framework have been defined, and assertions for library predicates (including the ones representing energy models) have been provided, the CiaoPP global static analysis can infer the resource usage of all the procedures in the program (as functions of input data sizes). A full description of how this is done can be found in [12].

Mode Analysis. It determines, for each argument in each predicate in the block representation, whether it acts as an input or an output argument. This is done using the (very accurate) information from the CiaoPP "sharing + freeness" (shfr) abstract domain. Accuracy is critical here to set up meaningful and solvable recurrence expressions for resource usage (as we will see later).

Size Measure Analysis. CiaoPP uses type information to decide which metric to use to express data sizes from a set of predefined metrics (e.g., the value of an integer, int, or the depth of a term, depth).

Type analysis of the Ciao IR program in Figure 3 (right hand side) infers that fact will be called with $R 0$ bound to an integer and $R 0 \_3$ a free variable, and will succeed with $R 0 \_3$ bound to an integer. Also, fact_aux will be called with the first two arguments bound to integers, and the rest free, and, upon success, all of them will be bound to integers. Given that information, the chosen metric for all the arguments will be int.

Size Analysis. It determines the relative sizes of variable bindings at different program points. The size analysis (as well as the resource usage analysis) is performed for each strongly-connected component of the control flow graph of the program in reverse topological order. For each clause, size relations are propagated to express each output data size as a function of input data sizes. For recursive functions this is done symbolically, creating a set of recurrence relations that will be solved to get a closed form function.

For our running example, the recurrence relations set up for the size of the output argument $R 0 \_3$ of fact as a function of the size of the input argument $R 0$ (denoted $\mathrm{fact}_{R 0 \_3}(R 0)$ ) as well as the corresponding one for fact_aux are:

$$
\begin{aligned}
& \operatorname{fact}_{R 0_{-} 3}(R 0)=\text { fact_aux }_{R 0_{-} 4}(0 \leq R 0, R 0) \\
& \operatorname{fact}_{\text {aux }} \operatorname{Ro\_ }_{4}(B, R 0)=\left\{\begin{array}{lr}
R 0 * \operatorname{fact}_{R 0_{-} 3}(R 0-1) & \text { if } B \text { is true (i.e., } 0 \leq R 0) \\
1 & \text { if } B \text { is false (i.e., } 0>R 0)
\end{array}\right.
\end{aligned}
$$

Solving it gives the closed form function: $\operatorname{fact}_{R 0_{-} 3}(R 0)=R 0$ !

Resource Usage Analysis. It uses the size information inferred by the size analysis to set up recurrence equations representing the resource usage of predicates, and computes bounds to their solutions. Remember that $c_{i}$ represents the energy cost of each instruction, taken from the energy model. Let $b_{e}$ denote the energy consumption function for a predicate (block) b. Then, the inferred equations for fact are:

$$
\operatorname{fact}_{e}(R 0)=\text { fact_aux }_{e}(0 \leq R 0, R 0)+c_{\text {entsp }}+c_{s t w}+c_{l d w}+c_{l d c}+c_{l s s}+c_{b f}
$$




$$
\operatorname{fact}_{\text {aux }}(B, R 0)=\left\{\begin{array}{rr}
\operatorname{fact}_{e}(R 0-1)+c_{b u}+2 c_{l d w}+c_{\text {sub }}+ & \text { if } B \text { is true } \\
+c_{b l}+c_{m u l}+c_{\text {retsp }} & \text { if } B \text { is false }
\end{array}\right.
$$

If we assume (for simplicity of exposition) that each instruction has unitary cost, i.e., $c_{i}=1$ for all $i$, we obtain the energy consumed by fact as a function of its input data size $(R 0):$ fact $_{e}(R 0)=13 R 0+8$

Note that using average values in the model implies that the energy function for the whole program inferred by the upper-bound resource analysis is an approximation of the actual upper bound (possibly below it). Thus, theoretically, to ensure that the analysis infers an upper bound, we need to use upper bounds as well in the energy models. However, such bounds would be very conservative, causing a loss in accuracy that would make the analysis impractical.

\section{Benchmarks, Results and Evaluation}

The aim of the experimental evaluation is to perform a first comparison of actual hardware energy measurements, in terms of accuracy, with the values obtained from both the low-level Instruction Set Simulation (ISS) model and the Static Resource Analysis (SRA) implemented within the CiaoPP framework, to obtain an early estimation of the feasibility of the approach. To this end, we describe a selection of currently analyzable benchmarks, the method by which data was collected, and an evaluation of the analysis framework accuracy vs. the low-level ISS model and hardware measurements.

Benchmarks. For this type of evaluation we use as benchmarks mainly small mathematical functions. The structure of these programs is either iterative or recursive, with their cost depending on the function argument. For such programs state of the art solvers can easily provide the cost functions, by solving the system of recurrence relations provided by the SRA framework. Table 1 shows the benchmarks used in this comparison, their execution behaviour in relation to each function's parameters, and the method by which their cost function was calculated. Also, some hand-solved examples have been provided in addition to those that were solved using SRA, both to compare a manual solution to SRA and to provide an additional set of data points that will, in the future, be solved automatically.

\begin{tabular}{|c|c|c|c|}
\hline Function name & Description & Energy function & Calculation \\
\hline $\operatorname{fact}(N)$ & Calculates $N$ ! & $26.0 N+19.4$ & \multirow{4}{*}{ SRA } \\
\hline fibonacci $(\mathrm{N})$ & $N$ th Fibonacci no. & $30.1+35.6 \phi^{N}+11.0(1-\phi)^{N}$ & \\
\hline $\operatorname{sqr}(\mathrm{N})$ & Computes $N^{2}$ & $103.0 N^{2}+205.8 N+188.32$ & \\
\hline poweroftwo (N) & Calculates $2^{N}$ & $62.4 \cdot 2^{N}-312.3$ & \\
\hline sumofdigits $(\mathrm{N})$ & Adds all digits in $\mathrm{N}$ & $84.4\left\lceil\log _{10} N\right\rceil-78.7$ & \multirow{3}{*}{ By hand } \\
\hline isprime $(\mathrm{N})$ & Checks if $\mathrm{N}$ is prime & $58.6 N-35.5$ & \\
\hline power (base, exp) & Calculates base $e^{e x p}$ & $6.3\left(\log _{2} \exp +1\right)+6.5$ & \\
\hline
\end{tabular}

Table 1. Description of benchmark functions used in experiments and their corresponding energy functions. 
Experimental method. Hardware energy readings were obtained by repeatedly executing a benchmark function over a 0.5 second period, $T$, collecting a set of power samples, $P$, whilst counting the number of executions, $N_{\mathrm{fn}}$. From this, the energy of a single function call, $E_{\mathrm{fn}}=\frac{\operatorname{mean}(P) \times T}{N_{\mathrm{fn}}}$ is calculated. This was performed using a similar method to the collection of energy model data described in Section 2, but was performed on separate hardware so as to de-couple modelling from testing.

ISS modelling involved simulating the same function a smaller number of times than on the hardware in order to keep simulation time adequately low. The instruction statistics were then processed in order to produce an energy figure, and then that figure divided by $N_{\mathrm{fn}}$ was used during ISS in order to extract the energy of a single call. The ISS modelling framework currently has a less efficient test loop than the hardware, potentially reducing accuracy for very short function calls. Similarly, if too few function calls are made during the simulation due to a long-executing function, overrun in the test time may skew low-level energy figures.

Static resource usage analysis was performed by evaluating the produced cost function for a given benchmark with respect to the input arguments, immediately providing the energy cost of a single function call.

Results. Table 2 provides an example of test data for the fact (factorial) function. The hardware (HW), low-level Instruction Set Simulation model (ISS), and Static Resource Analysis (SRA) model energy figures are compared. The relative error of ISS and SRA are compared with respect to the HW energy and normalised as such. The cost function provided for this particular example, demonstrates the relationship between the input parameter, $N$, and the SRA estimate of such a call. This, together with data for a number of further benchmarks are presented in graph form in Figure 5.

\begin{tabular}{|c|c|c|c|c|c|c|}
\hline \multirow{2}{*}{$\begin{array}{c}\text { SRA cost } \\
\text { function }(n J)\end{array}$} & \multirow{2}{*}{$N$} & \multirow{2}{*}{$\begin{array}{r}\text { HW measured } \\
\text { energy }(\mathrm{nJ})\end{array}$} & \multicolumn{2}{|c|}{ Calculated energy (nJ) } & \multicolumn{2}{|c|}{ Error vs. HW } \\
\hline & & & ISS & SRA & ISS & SRA \\
\hline \multirow{7}{*}{$26.0 N+19.4$} & 1 & 53.1 & 62.8 & 45.3 & 1.18 & 0.85 \\
\hline & 2 & 78.0 & 83.8 & 71.3 & 1.07 & 0.91 \\
\hline & 4 & 127.7 & 125.7 & 123.1 & 0.98 & 0.96 \\
\hline & 8 & 227.1 & 209.6 & 226.8 & 0.92 & 1.00 \\
\hline & 16 & 426.0 & 377.4 & 434.2 & 0.89 & 1.02 \\
\hline & 32 & 823.8 & 713.4 & 849.0 & 0.87 & 1.03 \\
\hline & 64 & 1690.5 & 1387.0 & 1678.4 & 0.82 & 0.99 \\
\hline
\end{tabular}

Table 2. Actual and estimated energy consumption for the fact(N) function over a range of $N$.

In Figure 5, hardware measured energy is compared directly to ISS and SRA energy predictions for the set of four benchmarks. The relative errors are also plotted. In all cases, the ISS model is seen to improve in accuracy as the input parameter $N$ increases, in line with the expected inaccuracies arising from inefficiencies in the modelling loop used in simulation, as described in the previous subsection. In the case of the poweroftwo function, time limitations prevent the ISS model from approximating the function above $N=13$, approaching which 

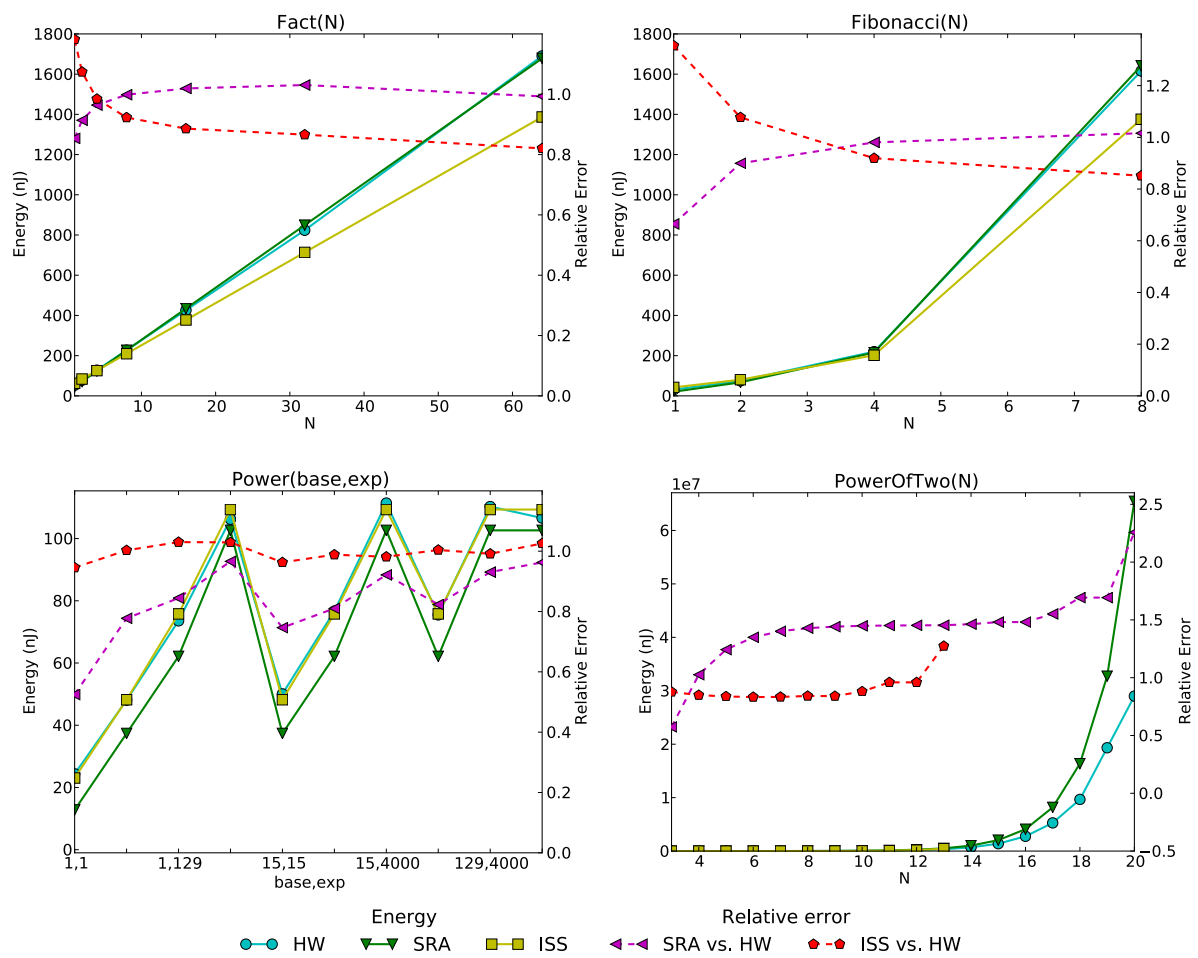

Fig. 5. HW energy, estimations and relative errors for (starting top-left, moving clockwise) fact, fibonacci, poweroftwo and power.

the error begins to increase markedly. The power function behaves in a similar way and demonstrates the relationship between multiple input arguments.

The SRA CiaoPP model does not suffer the same deficiencies, although it does incur a greater underestimation of energy for small values of $N$. The HW measurements unavoidably contain some loop code beyond the target function being examined and small $N$ values will increase the effects of this in the measurement. ISS in fact models this inefficiency directly, whereas SRA does not, hence the roughly symmetrical relative errors for the two models, particularly in the fact and fibonacci cases.

Both approaches are reliant on the same underlying instruction energy figures. Given that some instructions are not directly profiled and, instead, given an average value, accuracy is reduced when the distribution of instructions in a given program is such that the number of profiled instructions is low.

Overall, these results demonstrate both models' capabilities to estimate energy, with encouraging accuracy that can be improved upon. Further, the SRA approach is less restrictive, particularly in situations where simulation time might be prohibitively long. 


\section{Related Work}

There is not much work focusing on static analysis of energy consumption. The worst-case analysis presented in [8] distinguishes instruction-specific (not proportional to time, but to data) from pipeline-specific (roughly proportional to time) energy consumption.

The approach we have followed in this paper, as well as the one in [1], based on recurrence relations, derives from the seminal works on time analysis of [15] and [2]. A general framework to deal with user-defined resources was proposed later in [12]. A different approach is based on the potential method, such as in [7], which is based on a type-and-effect system. However, it is limited to polynomial bounds, which do not allow expressing some non-polynomial energy functions, as the ones we show in the experimental results table.

Transformation-based frameworks for program analysis that analyse low level microprocessor code [4] and Java source and bytecode $[1,11]$ have been proposed. In [1], cost relations are inferred directly for the bytecode programs, whereas in [11] the bytecode is first transformed into a Ciao program. Our transformation framework is closely related to [11] where the Jimple (a typed three-address code) representation of Java bytecode is transformed into Ciao. However, unlike Jimple, we employ transformations at lower level (XS1-ISA), irrespective of source language in general, where much of the program structure and typing information is trimmed away. Our transformation employs analysis techniques to reverse engineer ISA programs, which requires control flow graph reconstruction and transformation into an equivalent Ciao IR that safely approximates the semantics of the original ISA program.

Instruction Set Simulation can be used to estimate the energy of a program running on a suitably profiled hardware platform. Simple models for singlethreaded architectures have been demonstrated [13]. These have then been expanded upon, leading to models capable of modelling more complex hardware such as that used in this paper, which comprise a multi-threaded architecture [3].

\section{Conclusions and Future Work}

In this paper we introduce an approach for estimating the energy consumption of programs compiled for the XS1 architecture, based on a Horn clause transformation and the use of ISA level models that we have produced. We have shown the feasibility of the approach with a prototype implementation within the CiaoPP system, which has been successful in statically finding a good approximation of the energy consumed by a set of selected programs in our experiments.

The XS1 architecture is inherently multi-threaded, and the simulation-based model is able to provide energy estimates for this. Statically analysing multiple concurrent threads adds a significant new dimension of complexity to the modelling exercise. This is a goal of further work in order to provide meaningful analysis for contemporary multi-threaded programs running on this architecture.

Our analysis accuracy can also be further improved by propagating high-level program information such as types to the lower-level representations. We also intend to improve upon the energy measurements of commonly used instructions, which involves more complex techniques such as linear regression. This 
technique can also be used to construct energy models of intermediate compiler representations such as LLVM IR [9], which would enable us to apply our analysis techniques to more structured program representations. Another method for analysing LLVM IR would involve mapping low-level program instruction segments to LLVM IR segments and reusing the energy models at ISA level.

\section{References}

1. E. Albert, P. Arenas, S. Genaim, G. Puebla, and D. Zanardini. Cost Analysis of Java Bytecode. In Rocco De Nicola, editor, 16th European Symposium on Programming, ESOP'O\%, volume 4421 of Lecture Notes in Computer Science, pages 157-172. Springer, March 2007.

2. S. K. Debray and N. W. Lin. Cost Analysis of Logic Programs. ACM Transactions on Programming Languages and Systems, 15(5):826-875, November 1993.

3. Kerstin Eder and Steven Kerrison. Energy modelling and optimisation of software for a hardware multi-threaded embedded microprocessor. Technical report, University of Bristol, June 2013.

4. Kim S. Henriksen and John P. Gallagher. Abstract interpretation of PIC programs through logic programming. In Sixth IEEE International Workshop on Source Code Analysis and Manipulation (SCAM 2006), pages 184-196. IEEE Computer Society, 2006.

5. M. Hermenegildo, G. Puebla, F. Bueno, and P. López-García. Integrated Program Debugging, Verification, and Optimization Using Abstract Interpretation (and The Ciao System Preprocessor). Science of Computer Programming, 58(1-2), 2005.

6. M. V. Hermenegildo, F. Bueno, M. Carro, P. López, E. Mera, J.F. Morales, and G. Puebla. An Overview of Ciao and its Design Philosophy. Theory and Practice of Logic Programming, 12(1-2):219-252, January 2012.

7. Jan Hoffmann, Klaus Aehlig, and Martin Hofmann. Multivariate amortized resource analysis. ACM Trans. Program. Lang. Syst., 34(3):14, 2012.

8. Ramkumar Jayaseelan, Tulika Mitra, and Xianfeng Li. Estimating the worst-case energy consumption of embedded software. In IEEE Real Time Technology and Applications Symposium, pages 81-90. IEEE Computer Society, 2006.

9. Chris Lattner and Vikram S. Adve. LLVM: A compilation framework for lifelong program analysis and transformation. In $C G O$, pages 75-88, 2004.

10. David May. The xmos xs1 architecture. available online: http://www.xmos.com/published/xmos-xs1-architecture.

11. Mario Mendez-Lojo, Jorge A. Navas, and Manuel V. Hermenegildo. A flexible, (c)lp-based approach to the analysis of object-oriented programs. In LOPSTR, pages 154-168, 2007.

12. J. Navas, E. Mera, P. López-García, and M. Hermenegildo. User-Definable Resource Bounds Analysis for Logic Programs. In International Conference on Logic Programming (ICLP'07), Lecture Notes in Computer Science. Springer, 2007.

13. V. Tiwari, S. Malik, A. Wolfe, and M. T. C. Lee. Instruction level power analysis and optimization of software. In VLSI Design, 1996. Proceedings., Ninth International Conference on, pages 326-328, 1996.

14. Douglas Watt. Programming XC on XMOS Devices. XMOS Limited, 2009.

15. Ben Wegbreit. Mechanical program analysis. Commun. ACM, 18(9):528-539, 1975.

16. L. Xu, F. Sun, and Z. Su. Constructing Precise Control Flow Graphs from Binaries. University of California, Davis, Tech. Rep, 2009. 